\title{
Potential antibiotic resistant pathogens in community-acquired pneumonia: playing it safe is anything but
}

\author{
Grant W. Waterer ${ }^{1,2,3}$ \\ Affiliations: ${ }^{1}$ University of Western Australia, Perth, Australia. ${ }^{2}$ Northwestern University, Chicago, IL, USA. \\ ${ }^{3}$ Royal Perth Hospital, Perth, Australia.
}

Correspondence: Grant W. Waterer, Level 4, MRF Building, Royal Perth Hospital, GPO Box X2213, Perth 6847, Australia. E-mail: grant.waterer@uwa.edu.au

@ERSpublications

Do not think that using broad-spectrum antibiotics is safer! http://bit.ly/2Qu0PBf

Cite this article as: Waterer GW. Potential antibiotic resistant pathogens in community-acquired pneumonia: playing it safe is anything but. Eur Respir J 2019; 54: 1900870 [https://doi.org/10.1183/ 13993003.00870-2019].

Since the introduction of the concept of healthcare-associated pneumonia (HCAP) in the 2005 update on the treatment of nosocomial pneumonias by the American Thoracic Society and the Infectious Diseases Society of America [1], there has been considerable controversy over whether this new category has been helpful or harmful. The core of the problem is the tension between clinicians perceiving that missing a methicillin-resistant Staphylococcus aureus (MRSA) or Pseudomonas aeruginosa may have dire consequences for their patient and the goals of antibiotic stewardship in reducing the use of too broad spectrum antibiotics driving patient-specific side effects like renal toxicity, Clostridium difficile or vancomycin-resistant enterocoli [2] infection, and the more generalised harm through the promotion of antibiotic-resistant bacteria within the hospital environment.

There has been a significant increase in the use of anti-pseudomonal and anti-MRSA antibiotics as empirical treatment in the setting of community-acquired pneumonia (CAP) since the classification of HCAP was introduced [3]. This increase in use is despite an abundance of epidemiological data that these pathogens are actually extremely uncommon in the setting of CAP, outside of a few centres in the USA [2, 4-6].

There is no doubt that patients with HCAP risk factors have worse outcomes than patients with CAP, but this is not unexpected given the much higher rate of comorbidities in the former [7]. The key question is whether treating with anti-MRSA or anti-pseudomonal antibiotics has improved outcomes. Although mostly observational data, the evidence from published studies is that the use of broader spectrum antibiotic coverage in patients with HCAP risk factors does not improve outcomes [8-11], although this has not been a uniform finding $[12,13]$.

While the community-level harm from excessive broader spectrum antibiotic use is well acknowledged, clinicians have a tendency to think mainly of their individual patients (hence the need for antibiotic stewardship programmes). Therefore, although the evidence for using broad-spectrum therapy is underwhelming, clinicians concerned about the patient in front of them may still opt for broader spectrum therapy "just in case" on the basis that this is a safer approach. In this issue of the European Respiratory Journal, Wевв et al. [14] present data that should make clinicians rethink whether broader spectrum therapy is a safer option. 
In an observation cohort of nearly 2000 patients with pneumonia admitted to four hospitals in Utah, WЕвв et al. [14] compared the outcome of conventional therapy with broader spectrum therapy. Compared to previous retrospective studies, there was much better adjustment for severity at presentation, comorbidity and indication bias, aided by a comprehensive electronic medical record. Consistent with other studies already mentioned, they found that clinicians empirically treated nearly $40 \%$ of patients with anti-pseudomonal and/or anti-MRSA therapy yet only 3\% of patients had these pathogens identified. Interestingly, only about one-third of those treated with broad-spectrum empiric therapy had identifiable HCAP risk factors. After adjusting for comorbidities and indication bias, broad-spectrum antibiotic therapy was associated with a 4.6-fold increased risk of mortality, longer hospital stay and increased C. difficile infection.

While the above data argues strongly against broad-spectrum coverage, clinicians will still legitimately ask "but what about the patients who were not given empirical therapy that covered the pathogen"? Interestingly this scenario only arose in $1.4 \%$ of those treated with broad-spectrum therapy and $0.6 \%$ of those treated with standard therapy. In a case chart review of the 40 fatal cases, inadequate antibiotic therapy was not identified as a causative factor in any case. In contrast, in $17.5 \%$ of fatal cases there was an antibiotic-associated adverse event, usually due to piperacillin/tazobactam or vancomycin or both, suggesting that antibiotics may have been contributing factors in some deaths. This finding is entirely consistent with the now well-recognised substantial increase in neophrotoxicity with the combination of vancomycin and piperacillin/tazobactam $[15,16]$.

The study by Wевв et al. [14] is not a randomised, controlled trial, but it does put clinicians on notice that "playing it safe" by using broad-spectrum antibiotics may actually be "playing it risky". The only really robust predictor of MRSA or P. aeruginosa infection is prior culture [17-19], but there are individual centres where the prevalence is higher. It is extremely important that clinicians do not generalise findings from these high-prevalence centres to their own. There may be very specific circumstances where empirical therapy is warranted while samples are obtained and analysed (for example coverage of MRSA in a young adult with rapidly progressive pneumonia and severe sepsis in influenza season) but in general a treat "just in case" strategy is fundamentally flawed and almost certainly causing harm. There is no escaping the current reality that to practise good medicine clinicians must know the epidemiology of their local pneumonia pathogens and not use broad-spectrum antibiotics "just in case".

Conflict of interest: G.W. Waterer has nothing to disclose.

\section{References}

1 American Thoracic Society and Infectious Diseases Society of America. Guidelines for the management of adults with hospital-acquired, ventilator-associated, and healthcare-associated pneumonia. Am J Respir Crit Care Med 2005; 171: 388-416.

2 Webb BJ, Jones B, Dean NC. Empiric antibiotic selection and risk prediction of drug-resistant pathogens in community-onset pneumonia. Curr Opin Infect Dis 2016; 29: 167-177.

3 Tomczyk S, Jain S, Bramley AM, et al. Antibiotic prescribing for adults hospitalized in the etiology of pneumonia in the community study. Open Forum Infect Dis 2017; 4: ofx088.

4 Chalmers JD, Taylor JK, Singanayagam A, et al. Epidemiology, antibiotic therapy, and clinical outcomes in health care-associated pneumonia: a UK cohort study. Clin Infect Dis 2011; 53: 107-113.

5 Metersky ML, Frei C, Mortensen EM. Predictors of Pseudomonas versus methicillin-resistant Staphylococcus aureus in hospitalized patients with healthcare-associated pneumonia. Respirology 2016; 21: 157-163.

6 Restrepo MI, Babu BL, Reyes LF, et al. Burden and risk factors for Pseudomonas aeruginosa community-acquired pneumonia: a multinational point prevalence study of hospitalised patients. Eur Respir J 2018; 52: 1701190.

7 Bjarnason A, Asgeirsson H, Baldursson O, et al. Mortality in healthcare-associated pneumonia in a low resistance setting: a prospective observational study. Infect Dis (Lond) 2015; 47: 130-136.

8 Attridge RT, Frei CR, Pugh MJ, et al. Health care-associated pneumonia in the intensive care unit: Guideline-concordant antibiotics and outcomes. J Crit Care 2016; 36: 265-271.

9 Haessler S, Lagu T, Lindenauer PK, et al. Treatment trends and outcomes in healthcare-associated pneumonia. J Hosp Med 2017; 12: 886-891.

10 Rothberg MB, Zilberberg MD, Pekow PS, et al. Association of guideline-based antimicrobial therapy and outcomes in healthcare-associated pneumonia. J Antimicrob Chemother 2015; 70: 1573-1579.

11 Webb BJ, Dangerfield BS, Pasha JS, et al. Guideline-concordant antibiotic therapy and clinical outcomes in healthcare-associated pneumonia. Respir Med 2012; 106: 1606-1612.

12 Falcone M, Corrao S, Licata G, et al. Clinical impact of broad-spectrum empirical antibiotic therapy in patients with healthcare-associated pneumonia: a multicenter interventional study. Intern Emerg Med 2012; 7: 523-531.

13 Madaras-Kelly KJ, Remington RE, Sloan KL, et al. Guideline-based antibiotics and mortality in healthcare-associated pneumonia. J Gen Intern Med 2012; 27: 845-852.

14 Webb BJ, Sorensen J, Jephson A, et al. Broad-spectrum antibiotic use and poor outcomes in community-onset pneumonia: a cohort study. Eur Respir J 2019; 54: 1900057.

15 Burgess LD, Drew RH. Comparison of the incidence of vancomycin-induced nephrotoxicity in hospitalized patients with and without concomitant piperacillin-tazobactam. Pharmacotherapy 2014; 34: 670-676.

16 Gomes DM, Smotherman C, Birch A, et al. Comparison of acute kidney injury during treatment with vancomycin in combination with piperacillin-tazobactam or cefepime. Pharmacotherapy 2014; 34: 662-669. 
17 Torre-Cisneros J, Natera C, Mesa F, et al. Clinical predictors of methicillin-resistant Staphylococcus aureus in nosocomial and healthcare-associated pneumonia: a multicenter, matched case-control study. Eur J Clin Microbiol Infect Dis 2018; 37: 51-56.

18 Aliberti S, Reyes LF, Faverio P, et al. Global initiative for meticillin-resistant Staphylococcus aureus pneumonia (GLIMP): an international, observational cohort study. Lancet Infect Dis 2016; 16: 1364-1376.

19 Sibila O, Laserna E, Maselli DJ, et al. Risk factors and antibiotic therapy in P. aeruginosa community-acquired pneumonia. Respirology 2015; 20: 660-666. 